\title{
FOLIATIONS ON A SURFACE OF CONSTANT CURVATURE AND THE MODIFIED KORTEWEG-DE VRIES EQUATIONS
}

\author{
SHIING-SHEN CHERN \& KETI TENENBLAT
}

\author{
Dedicated to Professor Buchin Su on his 80 th birthday
}

\begin{abstract}
The modified $K d V$ equations are characterized as relations between local invariants of certain foliations on a surface of constant Gaussian curvature.
\end{abstract}

Consider a surface $M$, endowed with a $C^{\infty}$-Riemannian metric of constant Gaussian curvature $K$. Locally let $e_{1}, e_{2}$ be an orthonormal frame field and $\omega_{1}, \omega_{2}$ be its dual coframe field. Then the latter satisfy the structure equations

(1) $d \omega_{1}=\omega_{12} \wedge \omega_{2}, d \omega_{2}=\omega_{1} \wedge \omega_{12}, d \omega_{12}=-K \omega_{1} \wedge \omega_{2}$,

where $\omega_{12}$ is the connection form (relative to the frame field). We write

$$
\omega_{12}=p \omega_{1}+q \omega_{2}
$$

$p, q$ being functions on $M$.

Given on $M$ a foliation by curves. Suppose that both $M$ and the foliation are oriented. At a point $x \in M$ we take $e_{1}$ to be tangent to the curve (or leaf) of the foliation through $x$. Since $M$ is oriented, this determines $e_{2}$. The local invariants of the foliation are functions of $p, q$ and their successive covariant derivatives. If the foliation is unoriented, then the local invariants are those which remain invariant under the change $e_{1} \rightarrow-e_{1}$.

Under this choice of the frame field the foliation is defined by

$$
\omega_{2}=0 \text {, }
$$

and $\omega_{1}$ is the element of arc on the leaves. It follows that $p$ is the geodesic curvature of the leaves.

We coordinatize $M$ by the coordinates $x, t$, such that

$$
\omega_{2}=B d t, \quad \omega_{1}=\eta d x+A d t, \quad \omega_{12}=u d x+C d t,
$$

Received September 22, 1981. The first author is supported partially by NSF Grant MCS8023356. 
where $A, B, C, u$ are functions of $x, t$, and $\eta(\neq 0)$ is a constant. Thus the leaves are given by $t=$ const, and $\eta x$ and $u / \eta$ are respectiviely the arc length and the geodesic curvature of the leaves. Substituting (4) into (1), we get

$$
A_{x}=u B, \quad B_{x}=\eta C-u A, \quad C_{x}-u_{t}=-K \eta B .
$$

Elimination of $B$ and $C$ gives

$$
u_{t}=\left(\frac{A_{x}^{\prime}}{u}\right)_{x x}+\left(u A^{\prime}\right)_{x}+\eta^{2} K \frac{A_{x}^{\prime}}{u},
$$

where

$$
A^{\prime}=A / \eta
$$

By choosing

$$
A^{\prime}=-K \eta^{2}+\frac{1}{2} u^{2}
$$

we get

$$
u_{t}=u_{x x x}+\frac{3}{2} u^{2} u_{x}
$$

which is the modified Korteweg-de Vries ( $=M K d V$ ) equation.

Condition (8) on the foliation can be expressed in terms of the invariants $p, q$ as follows: By (2) and (4) we have

$$
u=\eta p, \quad C=A p+B q .
$$

If we eliminate $B, C$ in the second equation by using (5), it can be written

$$
\eta q=\left(\log \frac{A_{x}^{\prime}}{u}\right)_{x}=\left(\log p_{x}\right)_{x}
$$

Introducing the covariant derivatives of $p$ by

$$
d p=p_{1} \omega_{1}+p_{2} \omega_{2}, \quad d p_{1}=p_{11} \omega_{1}+p_{12} \omega_{2},
$$

we have

$$
p_{x}=p_{1} \eta, \quad p_{x x}=p_{11} \eta^{2} .
$$

Hence condition (11) can be written

$$
q=\left(\log p_{1}\right)_{1} .
$$

A foliation will be called a $K$-foliation, if (14) is satisfied. We state our result in

Theorem. The geodesic curvature of the leaves of a $K$-foliation satisfies, relative to the coordinates $x$, $t$ described above, an $M K d V$ equation.

The above argument can be generalized to $M K d V$ equations of higher order. The corresponding foliations are characterized by expressing $q$ as a function of $p, p_{1}, p_{11}, p_{111}, \cdots$. 
Is there a similar geometrical interpretation of the $K d V$-equation itself, which is

$$
u_{t}=u_{x x x}+u u_{x} ?
$$

We do not have a simple answer to this question. Unlike the $M K d V$-equation, the sign of the last term is immaterial, because it reverses when $u$ is replaced by $-u$. It is therefore of interest to know that by a different foliation and a different coordinate system one can be led to a $M K d V$-equation (9) where the last term has a negative sign.

For this purpose we put

$$
\omega_{2}=B d t, \quad \omega_{1}=v d x+E d t, \quad \omega_{12}=\lambda d x+F d t,
$$

where $\lambda$ is a parameter. Substitution into (1) gives

$$
F_{x}=-K v B, \quad B_{x}=-\lambda E+v F, \quad E_{x}-v_{t}=\lambda B .
$$

Suppose $K \neq 0$, we get, by eliminating $B, E$,

$$
v_{t}=\left(\frac{F_{x}^{\prime}}{K v}\right)_{x x}+\left(v F^{\prime}\right)_{x}+\frac{\lambda^{2}}{K v} F_{x}^{\prime},
$$

where

$$
F=F^{\prime} \lambda
$$

The choice

$$
F^{\prime}=\frac{K}{2} v^{2}-\lambda^{2}
$$

reduces (18) into

$$
v_{t}=v_{x x x}+\frac{3}{2} K v^{2} v_{x}
$$

Here the sign of the second term depends on the sign of $K$.

It can be proved that the choice (20) corresponds to a foliation which is characterized by

$$
q=\frac{p_{11}}{p_{1}}-3 \frac{p_{1}}{p}=\left(\log \frac{p_{1}}{p^{3}}\right)_{1}
$$

\section{References}

[1] S. S. Chern \& C. K. Peng, Lie groups and KdV equations, Manuscripta Math. 28 (1979) 207-217.

UNIVERSITY OF CALIFORNIA, BERKELEY

UNIVERSIDADE DE BRASILIA, BRASIL 
